\title{
Silicon Nanowire on Mirror Nanoantennas - \\ Engineering Hybrid Gap Mode for Light Sources and Sensing Platforms
}

Hiroshi Sugimoto, ${ }^{*,+,}$ Ryosuke Imaizumi, ${ }^{\dagger}$ Tatsuki Hinamoto, ${ }^{\dagger}$ Takahiro Kawashima, ${ }^{\S}$

\section{Minoru Fujiił}

†Department of Electrical and Electronic Engineering, Graduate School of Engineering, Kobe University, Rokkodai, Nada, Kobe 657-8501, Japan

†JST-PRESTO, Honcho 4-1-8, Kawaguchi, Saitama 332-0012, Japan

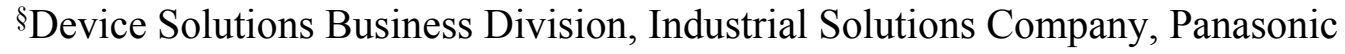

Corporation, 1006 Kadoma, Kadoma City, Osaka 571-8506, Japan

E-mail: sugimoto@eedept.kobe-u.ac.jp

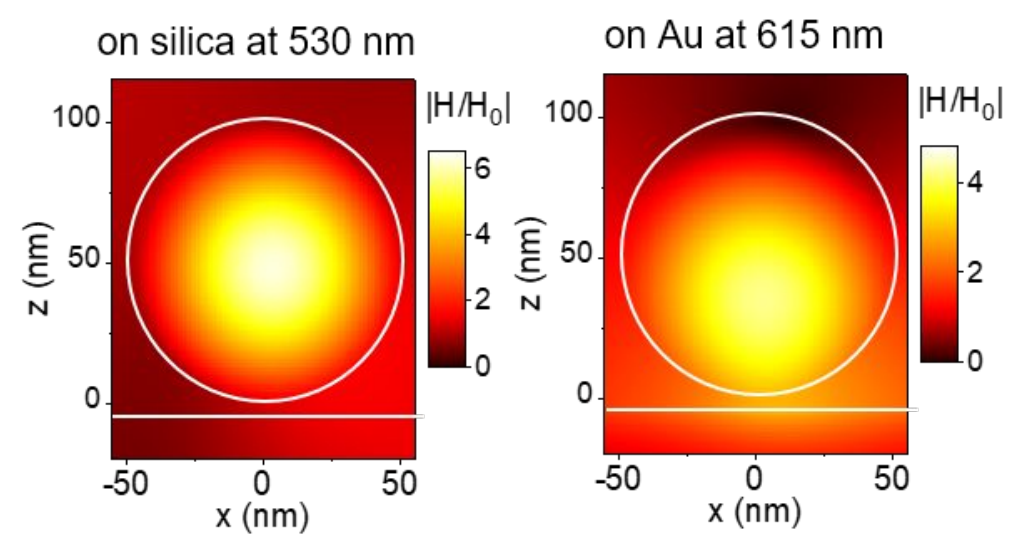

Figure S1. Magnetic field distribution of $\mathrm{Si} \mathrm{NW}-\mathrm{SiO}_{2}$ and $\mathrm{Si} \mathrm{NW}-\mathrm{Au}$ under p-polarized 
illumination.
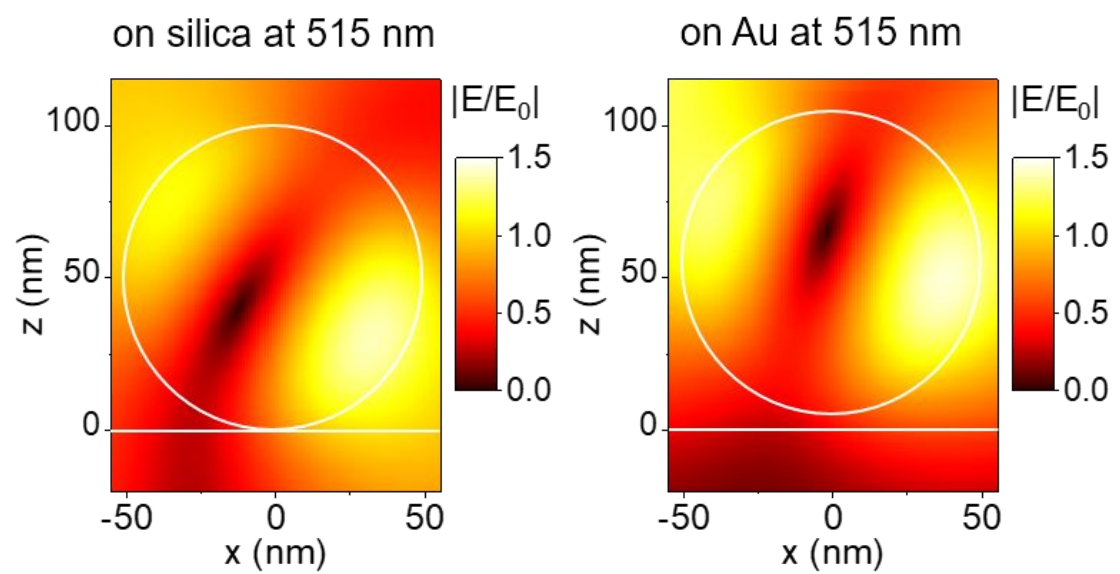

Figure S2. Electric field distribution of $\mathrm{Si} \mathrm{NW-SiO} 2$ and $\mathrm{Si} \mathrm{NW}-\mathrm{Au}$ under s-polarized illumination.

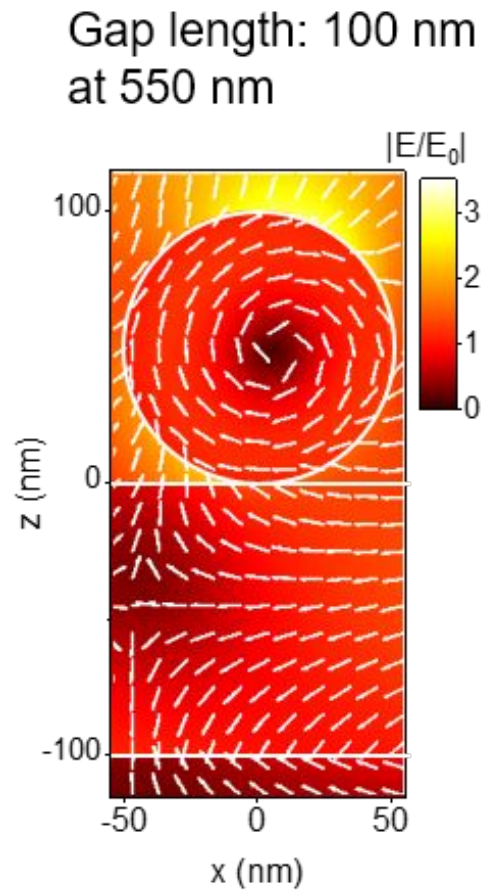

Figure S3. Electric field distribution of Si NW-Au with $100 \mathrm{~nm} \mathrm{SiO}_{2}$ spacer under TE excitation. 


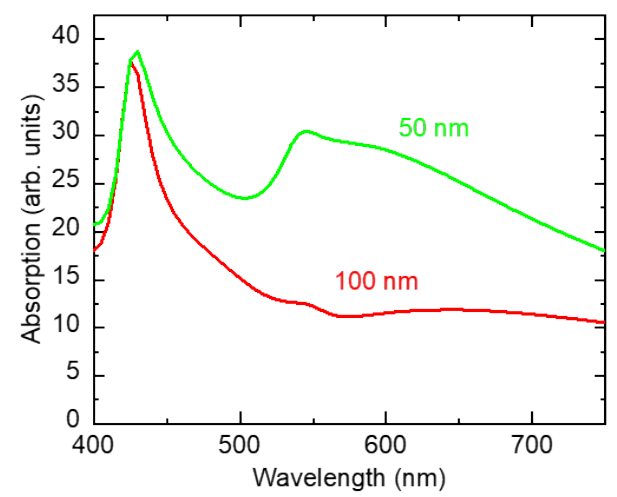

Figure S4. Absorption spectra of Si NW-Au with 50 (green) and $100 \mathrm{~nm}$ (red) $\mathrm{SiO}_{2}$ spacers.

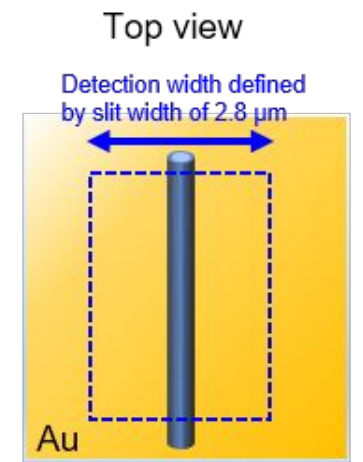

$$
\text { Cross-sectional view }
$$

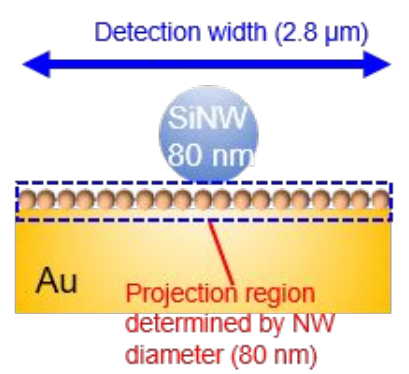

Figure S5. Schematic of the PL detection area and projection area of the NW for estimation of PL enhancement.

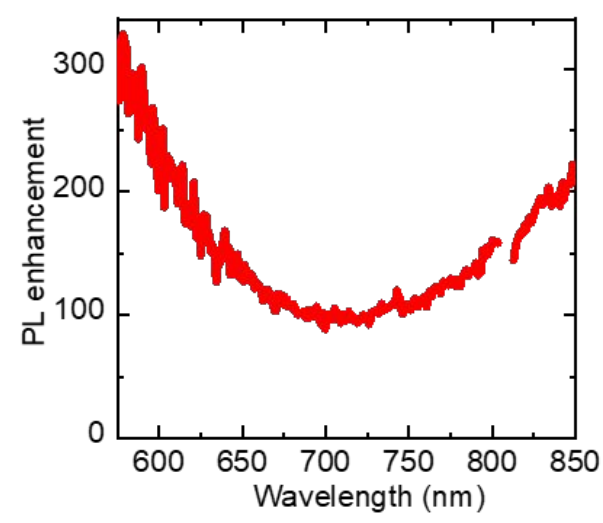

Figure S6. PL enhancement factor spectrum obtained using Eq.1 in the main text. 


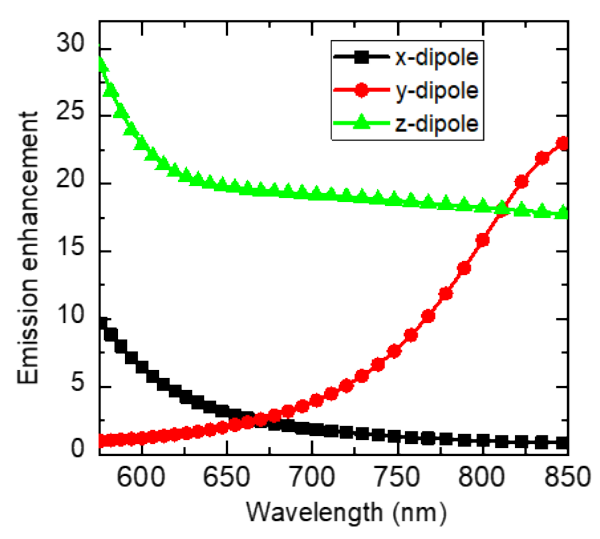

Figure S7. Emission enhancement spectra for dipoles oriented along three Cartesian coordinates.

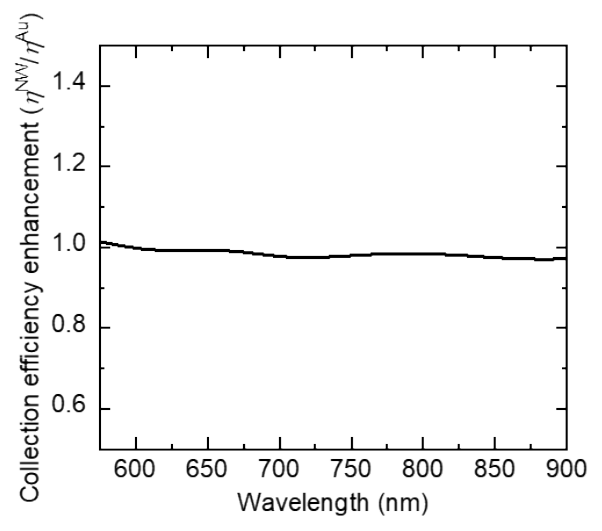

Figure S8. Collection efficiency enhancement $\left(\frac{\eta^{\mathrm{NW}}}{\eta^{\mathrm{Au}}}\right)$ with respect to QDs on Au. 